\title{
Density fluctuations from warm inflation
}

\author{
Chris Graham and Ian G. Mos:* \\ School of Mathematics and Statistics, Newcastle University, NE1 7RU, UK
}

(Dated: May 28, 2018)

\begin{abstract}
Thermal fluctuations provide the main source of large scale density perturbations in warm inflationary models of the early universe. For the first time, general results are obtained for the power spectrum in the case when the friction coefficient in the inflaton equation of motion depends on temperature. A large increase in the amplitude of perturbations occurs when the friction coefficient increases with temperature. This has to be taken into account when constructing models of warm inflation. New results are also given for the thermal fluctuations in the weak regime of warm inflation when the friction coefficient is relatively small.
\end{abstract}

\section{INTRODUCTION}

Observations of the cosmic microwave background have provided strong evidence that the primordial density fluctuations have a power spectrum very close to that predicted by inflationary models of the very early universe [1, 2, 3]. According to the standard theory, these density fluctuations can arise from quantum vacuum fluctuations during inflation [4]. Another possibility, which also fits the data, is that thermal fluctuations provide a source of density fluctuations [5, 6]. This is what happens in the warm inflationary scenario [7].

During an inflationary era particles are being produced continually but their density is also rapidly diminished by the expansion of the universe. What distinguishes warm inflationary models is that this particle production is sufficiently strong, compared to the effects of expansion, to produce a non-negligible particle density. Warm inflation occurs when the quantum statistical fluctuations in the particle number are large enough to influence the classical inflaton field and produce density fluctuations $[8,9]$.

The description of coupled fluctuations between classical sources and quantum radiation fields can be traced back to Einstein's classic discussion of a mirror immersed in black body radiation [10]. Stochastic fluctuations in the radiation field lead to fluctuations of the mirror. In an analogous way, fluctuations in the radiation field are transfered into inhomogeneities in the inflaton field. In a non-expanding universe, standard thermal field theory methods can be used to reduce the description of the system to a pair of coupled stochastic equations, one for the inflaton and one for the radiation density [11, 12]. The simplest case to analyse is where the radiation is close the thermal equilibrium, and then the particle production rate, the dissipation in the inflaton equation of motion and the source terms in the inflaton equation are all related by a single friction coefficient $\Gamma[11,12,13,14$.

There has been progress in extending the theory of thermal fluctuations to an expanding universe [15], but it seems that the basic equations which we require can be determined from the equivalence principle [8, 16]. This approach will be pursued further in Sect. III, where we present some new modifications to stochastic source terms in curved space. Our results, however, are broadly in line with those presented elsewhere which imply a close to scale-free power spectrum of density perturbations with small corrections depending on the slow-roll parameters [17, 18, 19].

The main objective in this paper is to present results for the power spectrum of fluctuations in inflationary models where the friction coefficient $\Gamma$ depends on the temperature $T$. Temperature dependence seems to be a common feature in explicit calculations of the particle production rates. In particular, there are high-temperature models of warm inflation with friction coefficient $\Gamma \propto T^{-1}$ [20, 21] and the two-stage decay models [22] with friction coefficient $\Gamma \propto T^{3}[20]$. High-temperature models are difficult to realise without producing large thermal corrections to the potential, which make the models unstable [23], and most consistent warm inflationary scenarios use the two-stage decay mechanism (see Ref. [7] for a review).

Individual models with temperature dependent friction coefficients have been examined using the numerical code written for Ref. [8], which solves coupled perturbation equations for the time evolution of the inflaton, radiation and metric. The model with $\Gamma \propto T^{-1}$ described in Ref. [8] produced an oscillatory power spectrum, but it has not been possible previously to formulate general laws for the functional dependence of the power spectrum.

An analysis of the coupled perturbation equations is presented in Sect. IV. We have been able to find approximate solutions to the perturbation equations on sub-horizon scales to determine general formulae for the power spectrum. These formulae have been checked against a numerical solution of the stochastic perturbation equations in section V.

*Electronic address: ian.moss@ncl.ac.uk 
The most important new results apply to the 'strong' regime of warm inflation where $\Gamma \gg 3 H$ and $H$ is the expansion rate. There is a large enhancement of the density perturbation amplitude when $\Gamma \propto T^{3}$, and a large diminution when $\Gamma \propto T^{-1}$. In practice, the power spectrum of density fluctuations is fixed by observation, and so the enhancement of the amplitude is equivalent to a reduction of the inflationary energy scale, and visa versa. We have also found that the oscillations of the power spectrum found in Ref. [8] are removed by the ensemble averaging. These results are an essential ingredient for the construction of warm inflationary models.

\section{WARM INFLATION}

We shall assume that particles are produced during the inflationary era and that the particle interactions are strong enough to produce a thermal gas of radiation with temperature $T$. The necessary conditions for this to happen where discussed in Ref. [13]. Warm inflation is said to occur when $T$ is larger than the energy scale set by the expansion rate $H$. The particle production is associated with a dissipative effect on the inflaton, whose equation of motion becomes 6. 24]

$$
\ddot{\phi}+(3 H+\Gamma) \dot{\phi}+V_{\phi}=0
$$

where $\Gamma(\phi, T)$ is a friction coefficient, $H$ is the Hubble parameter and $V_{\phi}$ is the $\phi$ derivative of the inflaton effective potential $V(\phi, T)$. The effectiveness of warm inflation can be parameterised by a parameter $r$, defined by

$$
r=\frac{\Gamma}{3 H}
$$

When $r \gg 1$ the warm inflation is described as being in the strong regime and when $r \ll 1$ the warm inflation is in the weak regime.

Consistent models of warm inflation [23] require a suppression of thermal corrections to the inflaton potential, so that the effective potential separates into inflaton and radiation components

$$
V(\phi, T)=V(\phi)+\rho_{r}(T)
$$

where $\rho_{r}$ is the radiation density

$$
\rho_{r}=\frac{\pi^{2}}{30} g_{*} T^{4}
$$

In this case, the time evolution is described by the equations

$$
\begin{aligned}
& \ddot{\phi}+(3 H+\Gamma) \dot{\phi}+V_{\phi}=0, \\
& \dot{\rho}_{r}+4 H \rho_{r}=\Gamma \dot{\phi}^{2}, \\
& 3 H^{2}=4 \pi G\left(2 V+2 \rho_{r}+\dot{\phi}^{2}\right)
\end{aligned}
$$

During inflation we apply a slow-roll approximation and drop the highest derivative terms in the equations of motion,

$$
\begin{aligned}
& 3 H(1+r) \dot{\phi}+V_{\phi}=0 \\
& 4 H \rho_{r}=\Gamma \dot{\phi}^{2} \\
& 3 H^{2}=8 \pi G V
\end{aligned}
$$

The validity of the slow-roll approximation depends on the slow roll parameters defined in [8],

$$
\epsilon=\frac{1}{16 \pi G}\left(\frac{V_{\phi}}{V}\right)^{2}, \quad \eta=\frac{1}{8 \pi G}\left(\frac{V_{\phi \phi}}{V}\right), \quad \beta=\frac{1}{8 \pi G}\left(\frac{\Gamma_{\phi} V_{\phi}}{\Gamma V}\right)
$$

The slow-roll approximation holds when $\epsilon \ll 1+r, \eta \ll 1+r$ and $\beta \ll 1+r$. Any quantity of order $\epsilon /(1+r)$ will be described as being first order in the slow roll approximation. 


\section{INFLATON FLUCTUATIONS}

Thermal fluctuations are the main source of density perturbations in warm inflation. Thermal noise is transfered to the inflaton field mostly on small scales. As the comoving wavelength of a perturbation expands, the thermal effects decrease until the fluctuation amplitude freezes out [9]. This may occur when the wavelength of the fluctuation is still small in comparison with cosmological scales.

In this section we shall review the equation for the inflaton fluctuations in the case where the friction coefficient is independent of temperature, i.e.

$$
\Gamma=\Gamma(\phi)
$$

We shall see how the stochastic inflaton dynamics transfers to an expanding universe and obtain new results for the case $\Gamma \ll 3 H$.

The behaviour of a scalar field interacting with radiation can be analysed using the Schwinger-Keldysh approach to non-equilibrium field theory [25, 26]. In flat spacetime, when the small-scale behaviour of the fields is averaged out, a simple picture emerges in which the field can be described by a stochastic system whose evolution is determined by a Langevin equation [27]. This takes the form

$$
-\nabla^{2} \phi(x, t)+\Gamma \dot{\phi}(x, t)+V_{\phi}=(2 \Gamma T)^{1 / 2} \xi(x, t)
$$

where $\nabla^{2}$ is the flat spacetime Laplacian and $\xi$ is a stochastic source. For a weakly interacting radiation gas the probability distribution of the source term can be approximated by a localised gaussian distribution with correlation function [11, 14],

$$
\left\langle\xi(x, t) \xi\left(x^{\prime}, t^{\prime}\right)\right\rangle=\delta^{(3)}\left(x-x^{\prime}\right) \delta\left(t-t^{\prime}\right)
$$

We shall restrict ourselves to this gaussian noise approximation.

We can use the equivalence principle to adapt the flat spacetime Langevin equation to an expanding universe during a period of warm inflation when $T>H$. The Langevin equation will retain its local form as long as the microphysical and thermal scales in the problem are small compared to the cosmological scale [7, 14]. However, the rest frame of the fluid will have a non-zero 3 -velocity $v_{r}^{\alpha}$ with respect to the cosmological frame and we must include an advection term. The Langevin equation becomes [16]

$$
\ddot{\phi}(x, t)+(3 H+\Gamma) \dot{\phi}(x, t)+\Gamma a^{-2} v_{r}^{\alpha} \partial_{\alpha} \phi(x, t)+V_{\phi}-a^{-2} \partial^{2} \phi(x, t)=\left(2 \Gamma_{\mathrm{eff}} T\right)^{1 / 2} \xi(x, t)
$$

where $a$ is the scale factor and $\partial^{2}$ is the Laplacian in an expanding frame with coordinates $x^{\alpha}$. The correlation function for the noise expressed in terms of the comoving cosmological coordinates becomes,

$$
\left\langle\xi(x, t) \xi\left(x^{\prime}, t^{\prime}\right)\right\rangle=a^{-3}(2 \pi)^{2} \delta^{(3)}\left(x-x^{\prime}\right) \delta\left(t-t^{\prime}\right) .
$$

A new parameter $\Gamma_{\text {eff }}$ has been introduced because at this stage we cannot determine the effects of the expansion on the noise term.

Next we treat the source term as a small perturbation and expand the inflaton field

$$
\phi(x, t) \rightarrow \phi(t)+\delta \phi(x, t)
$$

where $\delta \phi$ is the linear response due to the source $\xi$. This expansion is substituted into the Langevin equation and then we take the Fourier transform,

$$
\delta \ddot{\phi}(\mathbf{k}, t)+(3 H+\Gamma) \delta \dot{\phi}(\mathbf{k}, t)+V_{\phi \phi} \delta \phi(\mathbf{k}, t)+k^{2} a^{-2} \delta \phi(\mathbf{k}, t)=\left(2 \Gamma_{\mathrm{eff}} T\right)^{1 / 2} \xi(\mathbf{k}, t) .
$$

The inflaton will also generate metric inhomogeneities, but with a suitable choice of gauge, these can be discarded on sub-horizon scales (see [16]). We shall use a uniform expansion rate gauge. Eq. (18) applies on scales which are intermediate between the thermal averaging scale and the horizon scale. Later, we use a matching argument to extend the fluctuations to large scales.

The analysis of the Langevin equation can be simplified by introducing a new time coordinate $z=k /(a H)$ and using the slow roll parameters (11). We are led to the equation for $\delta \phi(\mathbf{k}, z)$,

$$
(1-\tilde{\epsilon})^{2} \delta \phi^{\prime \prime}-(3 r+2)(1-\tilde{\epsilon}) z^{-1} \delta \phi^{\prime}+\tilde{\epsilon}^{\prime} \delta \phi^{\prime}+3 \eta z^{-2} \delta \phi+\delta \phi=\left(2 \Gamma_{\mathrm{eff}} T\right)^{1 / 2}(1-\tilde{\epsilon})^{1 / 2} \hat{\xi}
$$


where a prime denotes a derivative with respect to $z$ and $\tilde{\epsilon}=\epsilon /(1+r)$. This reduces to

$$
\delta \phi^{\prime \prime}-(3 r+2) z^{-1} \delta \phi^{\prime}+\delta \phi=\left(2 \Gamma_{\mathrm{eff}} T\right)^{1 / 2} \hat{\xi}
$$

when we keep only the leading terms in the slow roll approximation. The noise term has been rescaled so that the correlation function is now

$$
\left\langle\hat{\xi}(\mathbf{k}, z) \hat{\xi}\left(\mathbf{k}^{\prime}, z^{\prime}\right)\right\rangle=(2 \pi)^{3} \delta^{(3)}\left(\mathbf{k}-\mathbf{k}^{\prime}\right) \delta\left(z-z^{\prime}\right) k^{-3}
$$

The perturbation equations can be solved using Green function techniques. The solution is

$$
\delta \phi=\int_{z}^{\infty} G\left(z, z^{\prime}\right)\left(z^{\prime}\right)^{1-2 \nu}\left(2 \Gamma_{\mathrm{eff}} T\right)^{1 / 2} \xi\left(z^{\prime}\right) d z^{\prime} .
$$

The retarded Green function $G\left(z, z^{\prime}\right)$ can be found in terms of Bessel functions,

$$
G\left(z, z^{\prime}\right)=\frac{\pi}{2} z^{\nu} z^{\prime \nu}\left(J_{\nu}(z) Y_{\nu}\left(z^{\prime}\right)-J_{\nu}\left(z^{\prime}\right) Y_{\nu}(z)\right) . \quad \text { for } z<z^{\prime}
$$

where

$$
\nu=\frac{3}{2}(1+r)=\frac{\Gamma+3 H}{2 H} .
$$

Corrections due to the time dependence of $\nu$ are similar in size to terms which we have already discarded in the slow roll approximation.

\section{A. Inflaton power spectrum}

The inflaton power spectrum $P_{\phi}(k, z)$ is defined by

$$
\left\langle\delta \phi(\mathbf{k}, z) \delta \phi\left(\mathbf{k}^{\prime}, z\right)\right\rangle=P_{\phi}(k, z)(2 \pi)^{3} \delta\left(\mathbf{k}+\mathbf{k}^{\prime}\right)
$$

Substituting the first order inflaton perturbation from (22) and the correlation function (21) gives

$$
P_{\phi}(k, z)=\left(2 \Gamma_{\mathrm{eff}} T\right) k^{-3} \int_{z}^{\infty} d z^{\prime} G\left(z, z^{\prime}\right)^{2}\left(z^{\prime}\right)^{2-4 \nu}
$$

in the slow-roll approximation.

Integrals of this type are examined in appendix A The large $z$ approximation corresponds to length scales much smaller than the horizon size, and we can use this to examine the flat space limit,

$$
P_{\phi}(k, z) \sim\left(2 \Gamma_{\mathrm{eff}} T\right) k^{-3} \frac{z}{4(\nu-1)}
$$

If the equivalence principal applies, then the result must agree with a calculation using flat space thermal field theory (where the physical momentum is $k a^{-1}$ ),

$$
P_{\phi}(k, z)=k a^{-1} T k^{-3}
$$

This allows us to read off the value of the constant $\Gamma_{\text {eff }}$ from a comparison of Eq. (27) and Eq. (28) using Eq. (24),

$$
\Gamma_{\text {eff }}=\Gamma+H
$$

The value of the coefficient is required for any analysis of the density perturbations in the weak regime $(r<1)$ of warm inflation. Such calculations have previously used the friction term $\Gamma+3 H$ for $\Gamma_{\text {eff }}$, but fortunately this has little effect on the final power spectrum [7].

When $r$ is small and $\nu \approx 3 / 2$, the perturbation amplitude at horizon crossing $(z=1)$ can be found numerically,

$$
P_{\phi}(k, 1) \approx 1.526 k^{-3} H T
$$


When $r$ is large, the perturbation amplitude at horizon crossing is governed by a saddle point in the integral (26), given according to eq. (A11) by,

$$
k=a \sqrt{\frac{3}{2}}\left(H\left(t_{F}\right) \Gamma\left(t_{F}\right)\right)^{1 / 2} .
$$

We call the time in eq. (31) the freezeout time $t_{F}$ for the mode $k$. The freezeout time always precedes the horizon crossing time. An analytic approximation to the integral valid for large values of $r$ is given in eq. (A5). With this we recover the result derived in [8], improving on an earlier results in [9],

$$
P_{\phi}(k, 1) \approx k^{-3} \frac{\sqrt{\pi}}{2}(\Gamma H)^{1 / 2} T .
$$

An approximation which works remarkably well for both large and small values of $r$ is given by [7]

$$
P_{\phi}(k, 1) \approx k^{-3} \frac{\sqrt{\pi}}{2} H^{1 / 2}(\Gamma+3 H)^{1 / 2} T .
$$

We shall compare the accuracy of this approximation to numerical results in Sect. V.

\section{COUPLED FLUCTUATIONS}

In this section we analyse the density perturbations in the case where the friction coefficient in the inflaton equation depends on temperature, i.e.

$$
\Gamma \equiv \Gamma(T, \phi)
$$

Temperature dependence in the friction coefficient is a feature of many models [20], and numerical calculations have shown that this can lead to interesting effects on the density perturbations [8]. The temperature dependence of the friction coefficient is parameterised by a coefficient $c$,

$$
c=\frac{T \Gamma_{T}}{\Gamma}
$$

The consistency of warm inflation requires only that $c<4$, and values of $c=3$ and $c=-1$ arise in calculations of the reheating term for different models.

The non-zero value of $c$ leads to additional terms in the fluctuation equations through

$$
\delta \Gamma=\Gamma_{T} \delta T+\Gamma_{\phi} \delta \phi
$$

The extra $\delta \phi$ terms depend on the slow-roll parameter $\beta$ and they are first order in the slow-roll approximation, but the $\delta T$ terms are leading order and couple the perturbations of the inflaton and the radiation.

\section{A. Radiation fluctuations}

There are two sources of fluctuations in the radiation. The first source is purely statistical and caused by the microscopic particle motions. These fluctuations can be described as photon number fluctuations [5], thermodynamic or quantum field fluctuations [8], but all approaches agree on the power spectrum $P_{s}$ for $\delta \rho_{r}$ in the flat space limit,

$$
P_{s}=4 \rho_{r} T a^{-3} \text {. }
$$

An estimate of the relative size of these fluctuations can be made by comparing the statistical fluctuations to the scalar field potential fluctuations $V_{\phi} \delta \phi$ calculated using Eq. (33) at horizon crossing $(k=a H)$,

$$
\frac{P_{s}}{V_{\phi}^{2} P_{\phi}} \sim \frac{\rho_{r} T a^{-3}}{V_{\phi}^{2} H^{1 / 2}(\Gamma+3 H)^{1 / 2} T k^{-3}} \sim \frac{r}{(1+r)^{3 / 2}}
$$

These stochastic radiation fluctuations are relatively unimportant in both the weak $(r \ll 1)$ and strong $(r \gg 1)$ warm inflationary regimes. 
The second source of fluctuations, which turns out to play the dominant role, is due to the inhomogeneous energy flux from the inflaton field. We can describe these fluctuations using first order perturbation theory. This can be formalated in many equivalent ways [8, 28], but the formulation we use here is based on Ref. [29], and Ref. [16]. The transfer of momentum and energy into the radiation is described by a momentum flux $J$ and an energy flux $\delta Q$, given by

$$
\begin{aligned}
J & =-\Gamma \dot{\phi} \delta \phi, \\
\delta Q & =\dot{\phi}^{2} \delta \Gamma
\end{aligned}
$$

If we keep only the leading terms in the slow roll approximation, the metric perturbations drop out and the energy density perturbations in a uniform expansion gauge satisfy the equation

$$
\delta \ddot{\rho}_{r}+9 H \delta \dot{\rho}_{r}+\left(20 H^{2}+\frac{1}{3} k^{2} a^{-2}\right) \delta \rho_{r}=k^{2} a^{-2} J+5 H \delta Q+\delta \dot{Q}
$$

The energy flux (40) can be expressed in terms of $\delta \rho_{r}$ using Eqs. (35) and (36) by

$$
\delta Q=c H \delta \rho_{r}
$$

The radiation fluctuations therefore satisfy

$$
\delta \ddot{\rho}_{r}+(9-c) H \delta \dot{\rho}_{r}+\left((20-5 c) H^{2}+\frac{1}{3} k^{2} a^{-2}\right) \delta \rho_{r}=-k^{2} a^{-2}(\Gamma \dot{\phi}) \delta \phi
$$

The equation for the inflaton fluctuations can simply be obtained by adding a term $\delta \Gamma \dot{\phi}$ to Eq. (18),

$$
\delta \ddot{\phi}+3 H(1+r) \delta \dot{\phi}+k^{2} a^{-2} \delta \phi+3 c r(\Gamma \dot{\phi})^{-1} \delta \rho_{r}=\left(2 \Gamma_{\mathrm{eff}} T\right)^{1 / 2} \xi
$$

There are now two coupled equations which we can use to determine the fluctuation power spectrum $P_{\phi}$.

An important feature of the coupled system is the growing mode $\delta \phi \propto a^{3 c}$ present for times such that $k \gg a H \gg k / r$. This replaces the constant mode responsible for freezing out the perturbation amplitude on sub-horizon scales for the uncoupled system. The concept of 'freezout' is a useful one nevertheless, as it defines the time when the noise terms leave their imprint on the final fluctuation amplitude. We will retain the terminology, but with the understanding that 'freezout' now means approaching the growing mode.

\section{B. Asymptotic expansions}

We shall determine the fluctuation amplitude for large $r$ by using a matched asymptotic expansion technique. To begin with, we convert the fluctuation equations to the variable $z=k /(a H)$ and drop the terms which are first order in the slow-roll approximation,

$$
\begin{aligned}
\delta \phi^{\prime \prime}-(3 r+2) z^{-1} \delta \phi^{\prime}+\delta \phi+c r z^{-2}(\Gamma \dot{\phi})^{-1} \delta \rho_{r} & =\left(2 \Gamma_{\mathrm{eff}} T\right)^{1 / 2} \hat{\xi} \\
\delta \rho_{r}^{\prime \prime}-(8-c) z^{-1} \delta \rho_{r}^{\prime}+(20-5 c) z^{-2} \delta \rho_{r}+\frac{1}{3} \delta \rho_{r}+(\Gamma \dot{\phi}) \delta \phi & =0
\end{aligned}
$$

We suppose that $r$ is a large parameter and $c r=O(r)$.

$$
\text { 1. The range } z<<r^{1 / 2}
$$

In the range $z<<r^{1 / 2}$, the stochastic source terms are relatively unimportant and the leading order contribution to Eq. (45) for large $r$ is simply

$$
\delta \rho_{r} \approx c^{-1}(\Gamma \dot{\phi}) z \frac{\partial}{\partial z} \delta \phi
$$

Substitute this into Eq. (46) to get

$$
z \frac{\partial}{\partial z}\left(z \frac{\partial}{\partial z}-5\right)\left(z \frac{\partial}{\partial z}+c-4\right) \delta \phi+\frac{1}{3} z^{2}\left(z \frac{\partial}{\partial z}+\frac{3 c}{2}\right) \delta \phi=0
$$


The solutions to this equation are Meijer- $G$ functions, (see [30]),

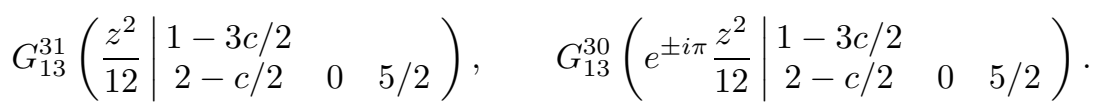

We are interested in the solution which grows most rapidly with time. Since the time evolution proceeds from large to small $z$, this is the solution which decays most rapidly with $z$.

The asymptotic expansion of the Meijer- $G$ functions for large $z$ can be found using standard techniques, in particular

$$
G_{13}^{31}\left(\frac{z^{2}}{12} \mid \begin{array}{ccc}
1-3 c / 2 & \\
2-c / 2 & 0 & 5 / 2
\end{array}\right) \sim\left(\frac{z^{2}}{12}\right)^{-3 c / 2} \Gamma_{R}(3 c / 2) \Gamma_{R}(3 c / 2+5 / 2) \Gamma_{R}(2+c)\left\{1-\frac{9 c(3 c+5)(c+2)}{z^{2}}+\ldots\right\}
$$

where $\Gamma_{R}$ is the Gamma function. When $c$ is positive, this is growing mode $\propto a^{3 c}$. For the other solutions

$$
G_{13}^{30}\left(e^{ \pm i \pi} \frac{z^{2}}{12} \mid \begin{array}{ccc}
1-3 c / 2 & \\
2-c / 2 & 0 & 5 / 2
\end{array}\right) \sim \sqrt{\pi}\left(\frac{z^{2}}{12}\right)^{(3+c) / 2} e^{\mp i z / \sqrt{3} \mp i \pi(c-1) / 2}
$$

These oscillatory solutions grow more rapidly with time than the power-law solutions when $c<-3 / 4$. We set

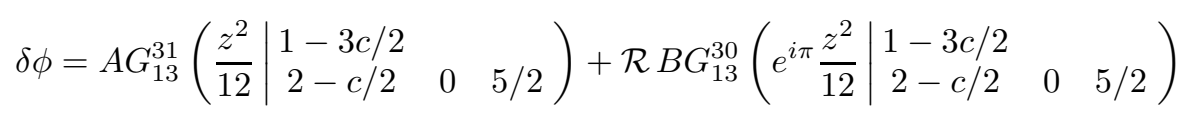

where $A$ and $B$ are constants and $\mathcal{R}$ indicates taking the real part.

\section{The range $1<<z<<r$}

In the range $1<<z<<r$ we have to take into account the noise term. If $c>-3 / 4$, then $\delta \rho_{r}$ has power-law behaviour and the leading terms in Eq. (46) give,

$$
\delta \rho_{r} \approx-3(\Gamma \dot{\phi}) \delta \phi
$$

Substitute into Eq. (45),

$$
\delta \phi^{\prime \prime}-(3 r+2) z^{-1} \delta \phi^{\prime}+\delta \phi-9 c r z^{-2} \delta \phi=(2 \Gamma T)^{1 / 2} \hat{\xi}
$$

As before, we solve this using a Green function,

$$
G^{c}\left(z, z^{\prime}\right)=\frac{\pi}{2} z^{\nu} z^{\prime \nu}\left(J_{\nu+3 c}(z) Y_{\nu+3 c}\left(z^{\prime}\right)-J_{\nu+3 c}\left(z^{\prime}\right) Y_{\nu+3 c}(z)\right) \quad \text { for } z<z^{\prime}
$$

where $\nu=3(1+r) / 2$. The power spectrum is given by

$$
P_{\phi}^{c \neq 0}(k, z)=(2 \Gamma T) k^{-3} \int_{z}^{\infty} d z^{\prime} G^{c}\left(z, z^{\prime}\right)^{2} z^{2-4 p}
$$

If we further restrict to $1<<z<<r^{1 / 2}$, then the integral can be approximated (see appendix A). Comparing to the previous result for $c=0$, we get

$$
\frac{P_{\phi}^{c \neq 0}(k, z)}{P_{\phi}^{c=0}(k, 1)} \approx \frac{\Gamma_{R}(3 c+3 / 2)}{\Gamma_{R}(3 / 2)} r^{3 c}\left(\frac{3}{z^{2}}\right)^{3 c}
$$

This result is not valid at horizon crossing $(z=1)$, and so a matching procedure is required. When $c<-3 / 4$ the solutions oscillate and Eq. (53) is no longer valid. The best we can do with a simple analysis is to conclude from Eq. (51) that $P_{\phi}^{c \neq 0}(k, z) \propto\left(z^{2} / r\right)^{3+c}$, multiplied by the ensemble average of oscillatory terms. We shall investigate this case numerically in the next section. 
3. Matching in the range $1<<z<<r^{1 / 2}$

For $c>-3 / 4$, both approximations are valid (and agree) in the the range $1<<z<<r^{1 / 2}$. Comparing $\delta \phi^{2}$ from Eq. (52) and $P_{\phi}$ from (57) gives $B=0$ and

$$
A^{2}=\left\{2^{3 c} \Gamma_{R}(3 c / 2) \Gamma_{R}(3 c / 2+5 / 2) \Gamma_{R}(2+c)\right\}^{-2} \frac{\Gamma_{R}(3 c+3 / 2)}{\Gamma_{R}(3 / 2)} r^{3 c} P_{\phi}^{c=0}(k, 1) .
$$

We are able to use Eq. (52) at $z=1$,

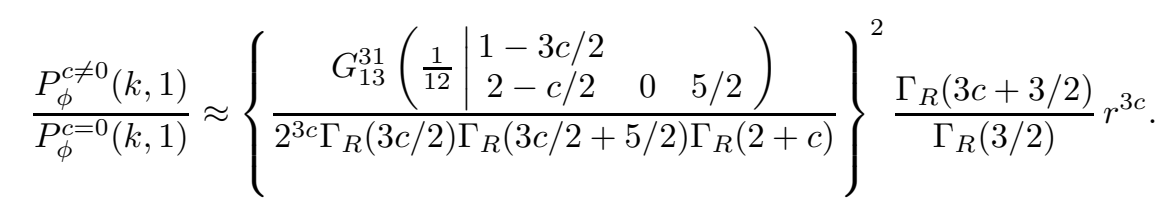

Numerical values of the result for different values of $c$ lie close to a simple power-law approximation

$$
\frac{P_{\phi}^{c \neq 0}(k, 1)}{P_{\phi}^{c=0}(k, 1)} \approx\left(\frac{r}{r_{c}}\right)^{3 c}
$$

where $r_{c}$ varies slowly in the range $0 \leq c \leq 3$, for example $r_{1} \approx 8.53, r_{2} \approx 7.66$ and $r_{3} \approx 7.27$.

\section{Damping effects}

Damping effects can suppress the radiation fluctuations and may be important when the thermal equilibrium of the radiation is imperfect. In warm inflation thermal equilibrium is disrupted by particle production from the evolving inflaton field which competes with the thermalising effects of self-interactions within the radiation gas [13]. The effectiveness of the thermalisation process can be parameterised by the value of $H \tau_{r}$, where $\tau_{r}$ is the relaxation time of the radiation.

The most important damping effect in a pure radiation gas comes from the shear viscosity $\eta$ [31], which modifies the perturbation equation (41) to,

$$
\delta \ddot{\rho}_{r}+\left(9 H+\eta k^{2} a^{-2}\right) \delta \dot{\rho}_{r}+\left(20 H^{2}+\frac{1}{3} k^{2} a^{-2}\right) \delta \rho_{r}=k^{2} a^{-2} J+5 H \delta Q+\delta \dot{Q}
$$

where 31]

$$
\eta=\frac{4}{15} \rho_{r} \tau_{r}
$$

The power-law behaviour of the growing mode is affected by the new damping term, so that a similar analysis to the undamped case now gives

$$
\frac{P_{\phi}^{c \neq 0}(k, 1)}{P_{\phi}^{c=0}(k, 1)} \approx\left(\frac{r}{r_{c}}\right)^{3 c^{\prime}}
$$

where

$$
c^{\prime}=\frac{2 c}{1+\left(1+24 c H \tau_{r} / 5\right)^{1 / 2}} .
$$

The equilibrium is maintained when $H \tau_{r} \ll 1$ and in this case $c^{\prime} \approx c$. However, the value of $c^{\prime}$ is still significant even when $H \tau_{r} \sim 1$ and the thermal equilibrium is very marginal. Enhancement of the fluctuations therefore appears to be a fairly robust phenomenon. 

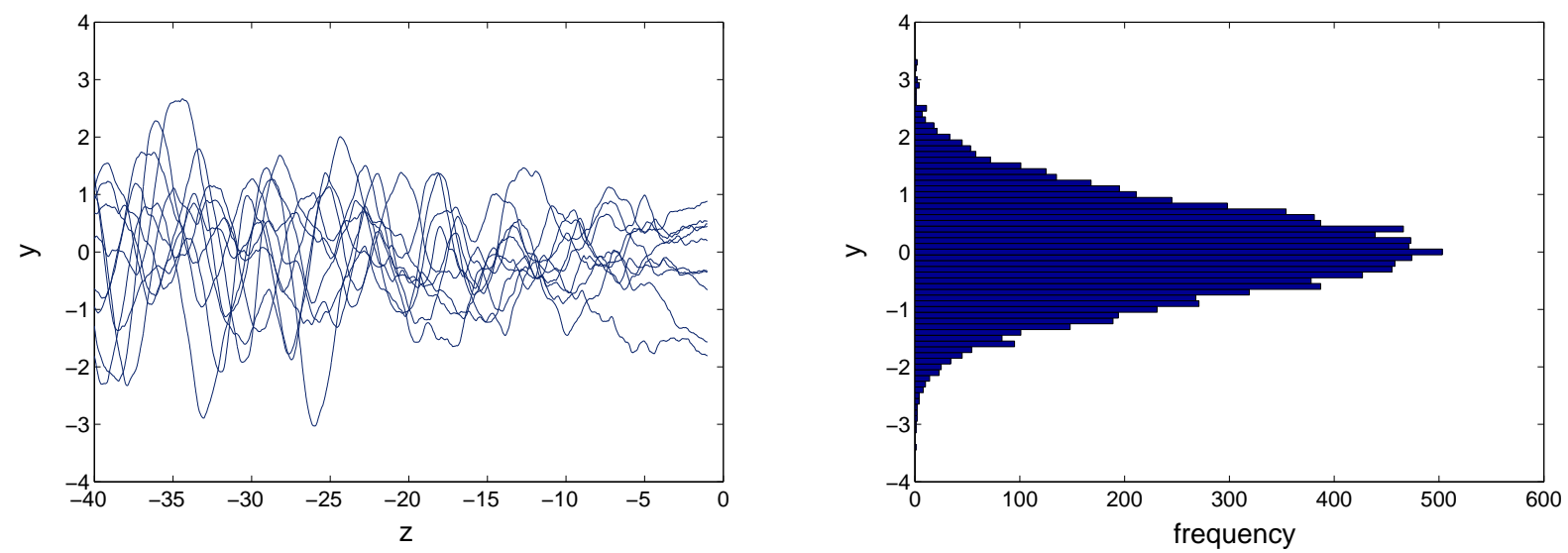

FIG. 1: The time evolution of the inflaton perturbation for several runs with $c=1$ and $r=5$ is shown on the left. The histogram on the right shows the values of the inflaton perturbation at horizon crossing $(z=-1)$ for 10000 runs.

\section{NUMERICAL SIMULATION}

The full set of perturbation equations for warm inflation, including the metric perturbations, where solved numerically by Hall et al. in ref. [8]. In the case where the parameter $c=-1$, the power-spectrum of the perturbations had a series of oscillations superimposed on the usual power-law behaviour in $k$.

The computer code used in ref. [8] uses the stochastic equations to set up the initial conditions and then evolves the full perturbation equations deterministically. The drawback with this approach is that the code only runs once for each mode, and does not necessarily produce the same results as an ensemble average of the stochastic system. In this section we shall solve stochastic equations for the perturbations, but we work to leading order in the slow-roll approximation and neglect the metric perturbations. The deterministic code helps provide solid evidence that the metric perturbations are small on sub-horizon scales, confirming the same conclusion obtained analytically in ref. [16].

We shall use rescaled variables

$$
y=k^{3 / 2}\left(2 \Gamma_{\mathrm{eff}} T\right)^{-1 / 2} \delta \phi, \quad w=k^{3 / 2}\left(2 \Gamma_{\mathrm{eff}} T\right)^{-1 / 2}(\Gamma \dot{\phi})^{-1} \delta \rho_{r} .
$$

The perturbation equations Eqs. (45) and (46) become

$$
\begin{array}{r}
y^{\prime \prime}-(3 r+2) z^{-1} y^{\prime}+y+c r z^{-2} w=\hat{\xi} \\
w^{\prime \prime}-(8-c) z^{-1} w^{\prime}+(20-5 c) z^{-2} w+\frac{1}{3} w+y=0
\end{array}
$$

where $\hat{\xi}$ is a normalised Gaussian random variable. The scalar field power spectrum is given by

$$
P_{\phi}=\left(2 \Gamma_{\text {eff }} T\right) k^{-3}\left\langle y(z)^{2}\right\rangle
$$

Departures from a scale-free spectrum arise from the dependence of $\Gamma, H$ and $T$ on the $k$-dependent horizon crossing (or freezeout) time. This can be determined from a numerical solution of the background homogeneous field equations. Our aim here is to examine how $\left\langle y(z)^{2}\right\rangle$ depends on $r$ and $c$.

Numerical results for $c=0, c=1$ and $c=-1$ are shown in figures 10.4 These have been obtained using a fourth order Runge-Kutta integration scheme and the Box-Muller algorithm for generating Gaussian random variables. Fig. 1 shows the time evolution of the inflaton field, plotted against $z=-k /(a H)$ for convenience. The histogram on the right shows the distribution of field values at horizon crossing $z=-1$ and fits a Gaussian distribution. The other graphs show $\left\langle y^{2}\right\rangle$ at horizon crossing for different values of $r=\Gamma / 3 H$.

The curves superimposed on the data are based on the theoretical results obtained in the previous section. For $c>-3 / 4$, Eq. (60) when converted into the rescaled variables corresponds to

$$
\left\langle y^{2}\right\rangle \approx \frac{1}{4}\left(\frac{\pi}{3}\right)^{1 / 2} r_{c}^{-3 c} r^{3 c-1 / 2} .
$$


The theoretical prediction is excellent for $c=0$ and $r \ll 1$. The data can also be approximated by rational fractions over the entire range, for example the approximation

$$
\left\langle y^{2}\right\rangle \approx \frac{\sqrt{3 \pi}}{4}_{\frac{(1+r)^{1 / 2}}{1+3 r}}
$$

works extremely well for $c=0$ and leads to the prediction for the inflaton power spectrum given in Eq. (33).

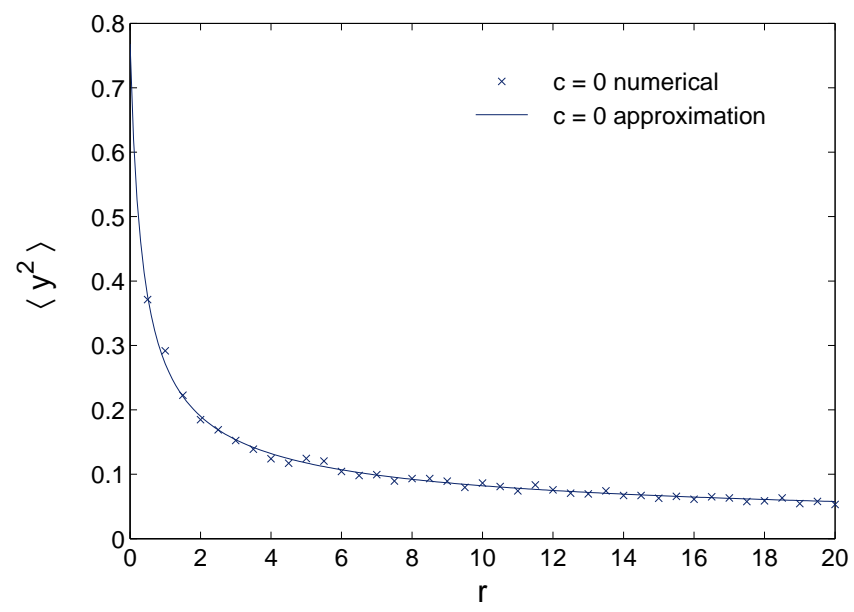

FIG. 2: The variance of the inflaton at horizon exit from the numerical simulations has been plotted against $r=\Gamma / 3 H$ for $c=0$ ( $\Gamma$ independent of $T$ ). The data are averages over 1000 runs. The curve shows the approximation Eq. (70).

The data for the $c=1$ case have been fit to a curve of the form $A_{1} r^{5 / 2}+B_{1} r^{3 / 2}$ based on the theoretical prediction. The need for next-to-leading order corrections to the asymptotic formula is indicated by the analysis, for example in Eq. (50). The coefficients for the best fit are shown in table I, and correspond to $r_{c}=8.2$ in Eq. (69), which is $5 \%$ from the theoretical prediction $r_{c}=8.5$. Results for $c=2$ and $c=3$ using the function $A_{c} r^{3 c-1 / 2}+B_{c} r^{3 c-3 / 2}$ are also shown in the table.

The most significant feature in the data for the $c=-1$ case is that we have found no evidence for the oscillations in the amplitude found previously in Ref. [8]. This is presumably due to the fact that the numerical code used in Ref. [8] only performs one run per mode, whereas we average over many runs. The results have been fit to a curve of the form $A_{-1} r^{-2}+B_{-1} r^{-3}$, in accordance with the leading order $\left(z^{2} / r\right)^{3+c}$ behaviour of $\delta \phi^{2}$ suggested in the previous section. The coefficients for the best fit are given in table 1

TABLE I: Coefficients in the numerical fits for the variance of the inflaton at horizon exit with their range of validity.

\begin{tabular}{llll}
\hline \hline$c$ & $A_{c}$ & $B_{c}$ & range \\
\hline 3 & $4.51 \times 10^{-9}$ & $3.34 \times 10^{-6}$ & $r>100$ \\
2 & $1.22 \times 10^{-6}$ & $4.39 \times 10^{-4}$ & $r>20$ \\
1 & $4.66 \times 10^{-4}$ & $2.94 \times 10^{-2}$ & $r>10$ \\
-1 & 5.30 & -37.1 & $r>20$ \\
\hline \hline
\end{tabular}




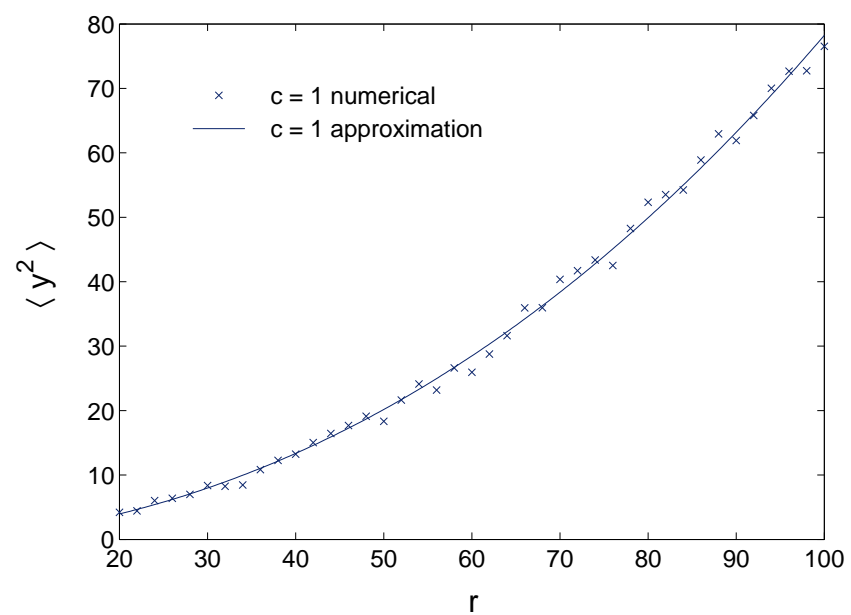

FIG. 3: The variance of the inflaton at horizon exit from the numerical simulations has been plotted against $r=\Gamma / 3 H$ for $c=1(\Gamma \propto T)$. The data are averages over 1000 runs. The curve shows the numerical fit $A_{1} r^{5 / 2}+B_{1} r^{3 / 2}$.

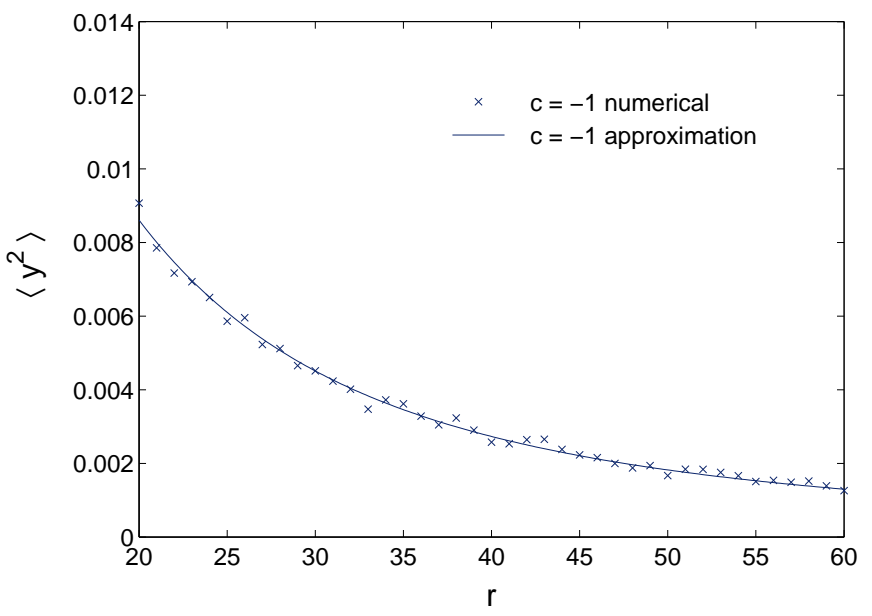

FIG. 4: The variance of the inflaton at horizon exit from the numerical simulations has been plotted against $r=\Gamma / 3 H$ for $c=-1\left(\Gamma \propto T^{-1}\right)$. The data are averages 1000 runs. The curve shows the numerical fit $A_{-1} r^{-2}+B_{-1} r^{-3}$.

\section{DENSITY FLUCTUATIONS}

We have seen that the small scale inflaton fluctuations freeze out by the time they cross the horizon. Whilst these fluctuations are freezing out, the metric fluctuations are relatively small (in the uniform expansion-rate gauge, for example). Eventually, the wavelength of the perturbations becomes far larger than the effective cosmological horizon and the metric perturbations become important. On these large scales it becomes possible to use a small-spatialgradient expansion (first formalised by Salopeck and Bond [32]). This approach allows us to define the curvature perturbation $\zeta$ so that it is conserved even in the non-linear theory [33, 34, 35]. The spectrum can be obtained, to high accuracy, by matching the small and large scale approximations at horizon crossing.

The fluctuations can be described entirely by the conserved expansion fluctuation $\zeta$ on constant density hypersurfaces [36], which is defined for general hypersurfaces by

$$
\zeta=\frac{1}{2} \ln (1+2 \varphi)+\frac{1}{3} \int \frac{d \rho}{p+\rho}
$$

where $\varphi$ is the spatial curvature perturbation. After using the slow roll equations,

$$
\zeta=\frac{1}{2} \ln (1+2 \varphi)+\int \frac{H}{\dot{\phi}} d \phi
$$


where $H \equiv H(\phi)$ and $\dot{\phi} \equiv \dot{\phi}(\phi)$ are given from eqs. (8, 10).

Consider a uniform curvature gauge $\varphi=0$ and $\zeta \equiv \zeta(\phi)$. When the inflaton perturbations are expanded as before in eq. (17), we have

$$
\zeta=\zeta_{\phi} \delta \phi
$$

where $\phi$ subscripts denote derivatives with respect to $\phi$ and $\zeta_{\phi}=H / \dot{\phi}$. Hence the power spectrum of density perturbations is

$$
P_{\zeta}(k)=\zeta_{\phi}^{2} P_{\phi}(k)
$$

In the previous section we evaluated the small scale inflaton perturbations in a uniform expansion-rate gauge, but on sub-horizon scales the metric perturbations are relatively small and the inflaton perturbations in the uniform expansion-rate gauge and the uniform curvature gauge are approximately equal.

In the strong regime of warm inflation we can use Eq (60) for the inflaton power spectrum when $r>r_{c}$ and $c>-3 / 4$, to give

$$
P_{\zeta}(k)=\frac{\sqrt{3 \pi}}{2} \frac{H^{3} T}{\dot{\phi}^{2}}\left(\frac{r}{r_{c}}\right)^{3 c} r^{1 / 2} k^{-3},
$$

This can be inverted, using the slow-roll equations (8,10), to express the basic energy scales of the inflationary model in terms of the fluctuation amplitude, $r$ and slow-roll parameters. The value of the potential when the mode with wave number $k$ crosses the horizon is given by

$$
V^{1 / 4}=g_{*}^{1 / 12} \epsilon^{1 / 4} r^{-3 / 4}\left(\frac{r}{r_{c}}\right)^{-c}\left(\frac{P_{\zeta}(k) k^{3}}{10^{-9}}\right)^{1 / 3}\left(3.73 \times 10^{15} \mathrm{GeV}\right),
$$

for $r>r_{c}$ and $c>-3 / 4$. The $c$ dependence depresses the energy scale of inflation relative to models with $c=0$. In most models this leads to an increase in $\Gamma / 3 H$ and $T / H$, making warm inflation stronger than in the $c=0$ case. When $c<0$ the energy scale of inflation increases and the warm inflation becomes weaker. On the other hand, if primordial tensor perturbations are seen in the cosmic microwave background, this would favour a large energy scale for inflation and $c<0$.

\section{CONCLUSION}

We have analysed the thermal fluctuations present in warm inflationary scenarios to obtain results for the primordial power spectrum using both analytical techniques and numerical simulations. For the first time it has been possible to present general formulae for the power spectrum in models where the fiction coefficient in the inflaton equation of motion depends on temperature. These results are crucial for building models of warm inflation which are consistent with cosmic microwave background observations.

The results for the primordial power spectrum of curvature fluctuations are as follows. For a friction coefficient $\Gamma$ which is independent of temperature, the familiar approximation

$$
P_{\zeta}(k)=\frac{\sqrt{\pi}}{2} \frac{H^{5 / 2}(\Gamma+3 H)^{1 / 2} T}{\dot{\phi}^{2}} k^{-3},
$$

fits both analytic and numerical calculations remarkably well for all values of $\Gamma$. This result can be applied whenever thermal fluctuations are the dominant source of curvature fluctuations, which is typically when $T>H$.

The power spectrum is given by a different formula when the friction coefficient depends on temperature. For $\Gamma \propto T^{c}$, and $r=\Gamma / 3 H \gg 1$, the amplitude is enhanced when $c>0$ and reduced when $c<0$. For accurate results we recommend using

$$
P_{\zeta}(k)=6 \frac{H^{3} T}{\dot{\phi}^{2}} f(r) k^{-3},
$$

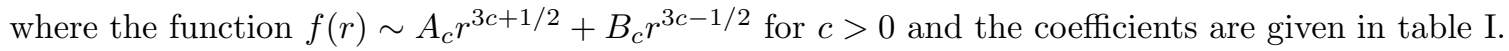

For less accurate work, a reasonable approximation for $c>0$ is provided by

$$
P_{\zeta}(k)=\frac{\sqrt{3 \pi}}{2} \frac{H^{3} T}{\dot{\phi}^{2}}(1+r)^{1 / 2}\left(1+\frac{r}{r_{c}}\right)^{3 c} k^{-3},
$$


with $r_{3} \approx 7.3$. This formula is consistent with the old result given by Eq. (77) for $c=0$ and approaches the numerical results for large $r$.

The most promising models of warm inflation to date use the 'two-stage' decay mechanism, for which the friction coefficient has $c=3[37,38]$. The new power spectrum is very different from the old result Eq. (77), and therefore these models must be analysed using the new power spectrum. The effect of the new formula is to reduce the inflationary scale, and it is likely that the restrictions on warm inflationary models will be relaxed.

It is known that warm inflationary models produce a significant amount of non-gaussianity, possibly enough to be observed in microwave background experiments [16, 39]. So far, the analysis of non-gaussianity has only been done in cases where the friction coefficient is independent of temperature. However, it seems possible that the analysis of the coupled perturbation equations initiated here could be extended to second order to obtain results for the non-gaussianity when the friction coefficient is temperature dependent.

\section{APPENDIX A: INTEGRALS}

We begin with an approximation to the integral

$$
F(z)=\int_{z}^{\infty} d z^{\prime} G\left(z, z^{\prime}\right)^{2}\left(z^{\prime}\right)^{2-4 \nu}
$$

where $\nu=3(1+r) / 2$ and the retarded Green function $G$ is given in eq. (23). The leading terms for large $\nu$ and fixed $z$, come from

$$
F(z) \approx \frac{\pi^{2}}{4} z^{2 \nu} Y_{\nu}(z)^{2} \int_{0}^{\infty} J_{\nu}\left(z^{\prime}\right)^{2} z^{2-2 \nu} d z^{\prime}
$$

This is a standard integral,

$$
\int_{0}^{\infty} J_{\nu}\left(z^{\prime}\right)^{2} z^{\prime 2-2 \nu} d z^{\prime}=\frac{\Gamma_{R}(\nu-1)}{4 \Gamma_{R}(2 \nu-1 / 2) \Gamma_{R}(\nu-1 / 2)}
$$

where $\Gamma_{R}$ is the gamma function. We also have

$$
z^{\nu} Y_{\nu}(z) \sim-\frac{2^{\nu}}{\pi \Gamma_{R}(\nu)}\left(1+\frac{z^{2}}{4 \nu}+\ldots\right) .
$$

Hence,

$$
F(z) \sim \sqrt{\frac{\pi}{32 \nu}}\left(1+\frac{z^{2}}{2 \nu}+\ldots\right) .
$$

A similar integral arises when we replace $G$ with the Green function $G^{c}$ in (55), and in this the case the integral, which we now call $F^{c}(z)$, has an asymptotic expansion with leading term

$$
F^{c}(z) \sim \frac{\Gamma_{R}(c+3 / 2)}{\Gamma_{R}(3 / 2)}\left(\frac{2 \nu}{z^{2}}\right)^{3 c} \sqrt{\frac{\pi}{32 \nu}} .
$$

in the range $z \ll \nu^{1 / 2}$.

A slightly more challenging problem is the integral

$$
F(z, g)=\int_{z}^{\infty} G\left(z, z^{\prime}\right)^{2} z^{\prime 2-4 \nu} g\left(z^{\prime}\right) d z^{\prime}
$$

where $g(z)$ is a smooth function. We proceed as above to get

$$
F(z, g) \approx \frac{\pi^{2}}{4} z^{2 \nu} Y_{\nu}(z)^{2} \int_{0}^{\infty} J_{\nu}\left(z^{\prime}\right)^{2} z^{\prime 2-2 \nu} g\left(z^{\prime}\right) d z^{\prime} .
$$

For large $\nu$ there is a Debye approximation for the Bessel functions which is valid in the range $0<z<\nu$,

$$
J_{\nu}(z) \sim(2 \pi \nu \tanh \alpha)^{-1 / 2} e^{\nu(\tanh \alpha-\alpha)}
$$


where $\nu \operatorname{sech} \alpha=z^{\prime}$. The relevant part of the integral becomes

$$
F(z, g) \approx \frac{\pi}{8} z^{2 \nu} Y_{\nu}(z)^{2} \nu^{2-2 \nu} \int_{0}^{\infty}(\cosh \alpha)^{2 \nu-3} e^{2 \nu(\tanh \alpha-\alpha)} g\left(z^{\prime}\right) d \alpha
$$

where $\nu \operatorname{sech} \alpha=z^{\prime}$. We find that there is a saddle point in this range at the value of $\alpha$ corresponding to $z=z_{F}$, where

$$
z_{F}=(3 \nu)^{1 / 2}
$$

Expanding about the saddle point gives

$$
F(z, g) \sim \sqrt{\frac{\pi}{32 \nu}} g\left(\tau_{F}\right)
$$

which agrees with our earlier result when $g(z)=1$. This saddle point is responsible for the phenomenon of 'freezing out' of the thermal fluctuations. The value of $z$ decreases with time and the fluctuations always freeze out before they cross the horizon at $z=1$.

[1] A. H. Guth, Phys. Rev. D 23, 347 (1981).

[2] A. Linde, Phys. Lett. 108B, 389 (1982).

[3] A. Albrecht and P. J. Steinhardt, Phys. Rev. Lett. 48, 1220 (1982).

[4] A. R. Liddle and D. H. Lyth, Cosmological inflation and large scale structure (Cambridge University Press, 2000).

[5] A. Berera and L. Z. Fang, Phys. Rev. lett. 74, 1912 (1995).

[6] I. G. Moss, Phys. lett. 154B, 120 (1985).

[7] A. Berera, I. G. Moss, and R. O. Ramos, Rept. Prog. Phys. 72, 026901 (2009), 0808.1855.

[8] L. M. H. Hall, I. G. Moss, and A. Berera, Phys. Rev. D69, 083525 (2004), astro-ph/0305015.

[9] A. Berera, Nucl. Phys B 585, 666 (2000).

[10] J. Stachel, ed., The collected papers of Albert Einstein (McGraw-Hill, New York, 1953), vol. 2, pp. 170-82, 206-22.

[11] M. Gleiser and R. O. Ramos, Phys. Rev. D 50, 2441 (1994).

[12] A. Berera, M. Gleiser, and R. O. Ramos, Phys. Rev. D 58, 123508 (1998).

[13] I. G. Moss and C. M. Graham, Phys. Rev. D78, 123526 (2008), 0810.2039.

[14] A. Berera, I. G. Moss, and R. O. Ramos, Phys. Rev. D76, 083520 (2007), 0706.2793.

[15] A. Berera and R. O. Ramos, Phys. Rev. D71, 023513 (2005), hep-ph/0406339.

[16] I. G. Moss and C. Xiong, JCAP 0704, 007 (2007), astro-ph/0701302.

[17] A. N. Taylor and A. Berera, Phys. Rev. D 62, 083517 (2000).

[18] H. P. De Oliveira, Phys. Lett. B526, 1 (2002), gr-qc/0202045.

[19] L. M. H. Hall and H. V. Peiris, JCAP 0801, 027 (2008), 0709.2912.

[20] I. G. Moss and C. Xiong (2006), hep-ph/0603266.

[21] A. Hosoya and M. A. Sakagami, Phys. Rev. D 29, 2228 (1984).

[22] A. Berera and R. O. Ramos (2003), hep-ph/0308211.

[23] I. G. Moss and C. Xiong, JCAP 0811, 023 (2008), 0808.0261.

[24] A. Berera, Phys. Rev. Lett. 75, 3218 (1995).

[25] J. Schwinger, J Math Phys 2, 407 (1961).

[26] L. V. Keldysh, Zh. Eksp. Teor. Fiz. 47, 1515 (1964).

[27] E. Calzetta and B. L. Hu, Phys. Rev. D 37, 2878 (1988).

[28] H. P. De Oliveira and S. E. Joras, Phys. Rev. D64, 063513 (2001), gr-qc/0103089.

[29] J.-C. Hwang and H. Noh, Class. Quantum Grav. 19, 527 (2002).

[30] A. Erdelyi, Higher Transcendental Functions, vol. 3 (McGraw-Hill, New York, 1953).

[31] S. Weinberg, Gravitation and Cosmology (Wiley, 1972).

[32] D. S. Salopek and J. R. Bond, Phys. Rev. D42, 3936 (1990).

[33] M. Sasaki and E. D. Stewart, Prog. Theor. Phys. 95, 71 (1996), astro-ph/9507001.

[34] D. H. Lyth, K. A. Malik, and M. Sasaki, JCAP 0505, 004 (2005), astro-ph/0411220.

[35] D. H. Lyth and Y. Rodriguez, Phys. Rev. Lett. 95, 121302 (2005), astro-ph/0504045.

[36] J. M. Bardeen, Phys. Rev. D22, 1882 (1980).

[37] M. Bastero-Gil and A. Berera, Phys. Rev. D76, 043515 (2007), hep-ph/0610343.

[38] M. Bastero-Gil and A. Berera, Int. J. Mod. Phys. A24, 2207 (2009), 0902.0521.

[39] I. G. Moss and C. M. Graham, JCAP 0711, 004 (2007), 0707.1647. 